\title{
Corticosteroids in severe pneumonia
}

\author{
O. Sibila* ${ }^{*, \#,+}$, C. Agustí ${ }^{\tau^{,+}}$and A. Torres ${ }^{\tau,+}$
}

ABSTRACT: The mortality rate in severe community- or hospital-acquired pneumonia is very high, ranging $20-50 \%$. Despite advances in antimicrobial therapy and supportive measures, this rate has not changed in recent years, suggesting that other factors are also responsible for the poor outcome. An abnormal increase in the local and systemic inflammatory response is associated with poor outcome, and this occurs despite adequate antibiotic therapy.

There is evidence that acute administration of corticosteroids decreases the inflammatory response and might decrease mortality in severe pneumonia. This has been shown in one small randomised controlled study, terminated prematurely due to $0 \%$ mortality in the intervention arm. In addition, an experimental study showed that glucocorticosteroids decrease lung inflammatory response and lung bacterial burden, confirming the results obtained through in vitro investigations.

Although these results are promising and suggest a novel role of glucocorticosteroids in pneumonia, the inherent risks and potential side-effects of these drugs require further controlled clinical trials in order to better define the target population before their general use in clinical practice. Specifically, dosage, period of administration, titration, tapering and side-effects are some of the key questions that need to be investigated.

KEYWORDS: Community-acquired pneumonia, corticosteroids, inflammatory response, nosocomial pneumonia, severe pneumonia

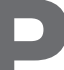
neumonia is the most prevalent infectious respiratory disease. It entails high morbidity and mortality and large healthcare system expenses. Community-acquired pneumonia (CAP) is one of the 10 leading causes of death worldwide. Approximately $20 \%$ of CAP patients require hospitalisation, $25 \%$ of whom are admitted to an intensive care unit (ICU) and have a mortality rate of $30-50 \%$ [1].

Despite progress in life-support measures and antimicrobial therapy, the mortality of severe pneumonia has not varied since the mid-1990s [2, 3], suggesting that other factors are of crucial importance in the evolution of this respiratory infection.

One of the key factors determining the progression of pneumonia is the host inflammatory response, which seems to be increased excessively in nonresponding severe pneumonia $[4,5]$. The purpose of the present article is to review the evidence in the literature supporting a beneficial role of glucocorticosteroids (GCs) as an adjuvant treatment in severe pneumonia.

\section{INFLAMMATORY RESPONSE ASSOCIATED WITH PNEUMONIA}

It is well known that the arrival of pathogens in the alveolar space creates a complex inflammatory response, with the interaction of several defence mechanisms and the production of a number of inflammatory mediators and acute phase proteins. The aim of this inflammatory response is to control the progression of the infection and to destroy microorganisms, and consists of several pro-inflammatory (tumour necrosis factor (TNF)- $\alpha$ and interleukin (IL)-1 $\beta,-6$ and -8) and antiinflammatory cytokines (IL-10, IL-1 receptor antagonist and the soluble $55-\mathrm{kDa}$ and $75-\mathrm{kDa}$ TNF receptors). Cytokines promote the migration of defence cells, such as neutrophils, lymphocytes and platelets, through the circulatory system to inflammatory sites [6].

All of this process is beneficial as long as it is limited to the control of local infection. If this reaction is overproportioned, several systemic consequences negatively influence the clinical progression of the infection [7].

This excessive inflammatory response is associated with the release of inflammatory mediators that can be detected in serum or bronchoalveolar lavage fluid (BALF) and have been shown to be of diagnostic and prognostic value.

The association between the overproduction of pro-inflammatory cytokines, such as TNF- $\alpha$ or
AFFILIATIONS

*Respiratory Dept, Son Dureta University Hospital, Palma de Mallorca, and ${ }^{\text {\#C} C a u b e t-C i m e r a ~ F o u n d a t i o n ~}$ Bunyola, and

"Respiratory Dept, Institut Clínic del Tòrax, Hospital Clínic, Institut d'Investigacions Biomèdiques August $\mathrm{Pi}$ i Sunyer, Barcelona, and ${ }^{+}$Ciber Enfermedades Respiratorias, Spain.

CORRESPONDENCE

A. Torres

Servei de Pneumologia Institut Clinic del Tórax Hospital Clinic Villarroel 170 08036 Barcelona Spain

Fax: 34932275549

E-mail: atorres@ub.edu

Received:

November 162007 Accepted after revision: February 182008

STATEMENT OF INTEREST None declared. 
IL-6, in serum and fatality in septic shock patients has confirmed that cytokines are markers of severity and probably major mediators involved in the pathogenesis of septic shock [8]. Studies concerning severe pneumonia have shown that, despite the inflammatory response initially being compartmentalised [9], an increase in inflammatory cytokine levels in serum is also detected and related to poor prognosis [10-13].

In addition, a recent study by YENDE et al. [14] has demonstrated that patients with high levels of circulating inflammatory cytokines in clinical stability (prior to infection) have a higher risk of suffering from CAP, suggesting a crucial role of the inflammatory response in all aspects of the pathogenicity of this infection.

\section{GLUCOCORTICOSTEROIDS AND THE INFLAMMATORY RESPONSE: MOLECULAR MECHANISMS}

GCs inhibit the expression and action of many cytokines involved in the inflammatory response associated with pneumonia. GCs are mainly transported in the blood complexed to transcortin (or corticosteroid-binding globulin) and albumin, although a small proportion is in a free metabolically activate state. The free GC molecules readily cross the plasma membrane into the cytoplasm. Once in the cytoplasm, GCs bind to their specific receptor, the GC receptor (GR) $\alpha$. GR $\alpha$ exists as a heterocomplex located in the cytoplasm of nearly all human cells [15]. The anti-inflammatory and immunosuppressive effects of GCs are due to three molecular mechanisms [15, 16]. First, the ligand-activated GR $\alpha$ binds as a homodimer to specific DNA sequences, so-called GR-responsive elements, usually located in the promoter regions of target genes, in order to induce transcription, a phenomenon called transactivation. Secondly, indirect negative regulation of gene expression (transrepression) is achieved by GR-protein interaction. The ligand-activated receptor binds as a monomer to key proinflammatory transcription factors, such as activator protein-1 and nuclear factor $-\kappa B$. The resulting complex inhibits the initiation of transcription of relevant genes. This mechanism has been shown to be involved in the inhibition of different gene products that play a central role in inflammation (table 1). The third mechanism is GC signalling through membrane-associated receptors and second messengers (so-called nongenomic pathways). The best-described nongenomic mechanism involves the activation of endothelial nitric oxide synthetase (eNOS). Nontranscriptional activation of eNOS by GR represents a physiologically important signalling pathway by which GCs exert their rapid anti-inflammatory effects. Binding of GCs to the GR stimulates phosphatidylinositol-3'-kinase and Akt kinase, leading to eNOS activation and nitric oxide-dependent vasorelaxation [17].

\section{ROLE OF GLUCOCORTICOSTEROIDS IN SEVERE PNEUMONIA}

Based on the critical role of the inflammatory response in the progression of infections and the anti-inflammatory potential of GCs, it is not surprising that these drugs have been tested in clinical practice. To date, the results have been controversial, and clear indications for their use are currently sparse. The usefulness of GCs seems to have been demonstrated in infections such as bacterial meningitis and pneumonia caused by Pneumocystis jiroveci [18]. In the field of sepsis, the crucial

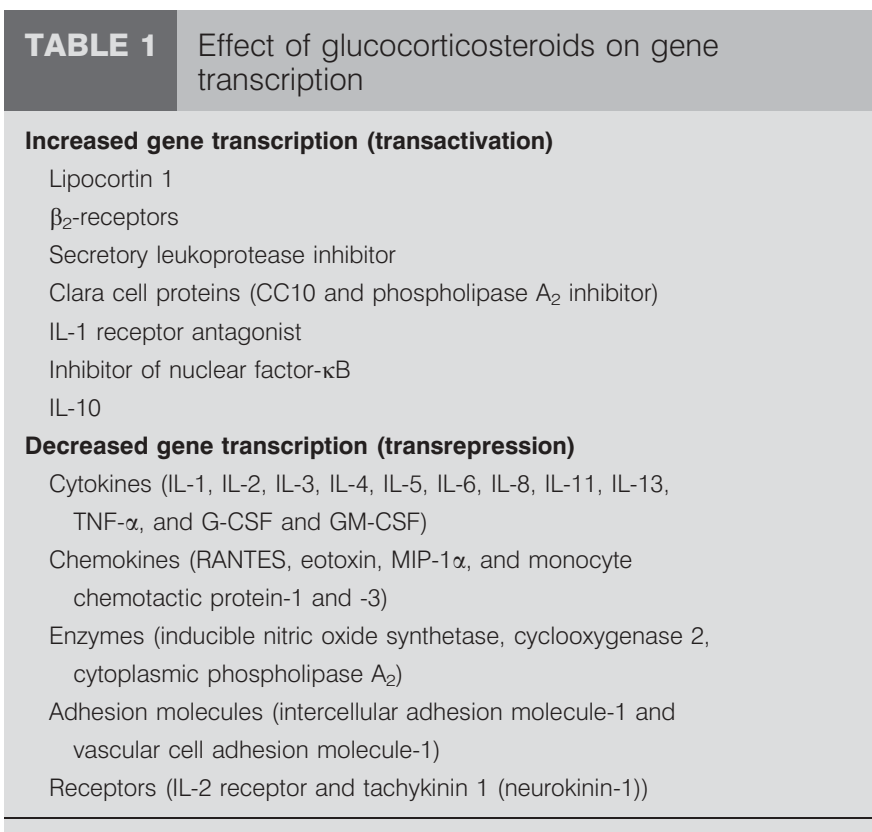

CC10: Clara cell 10-kDa protein; IL: interleukin; TNF- $\alpha$ : tumour necrosis factor- $\alpha$; G-CSF: granulocyte colony-stimulating factor; GM-CSF: granulocytemacrophage colony-stimulating factor; RANTES: regulated on activation normal T-cell expressed and secreted; MIP: macrophage inflammatory protein

role of the associated inflammatory response to infection has been well demonstrated. In this critical infection, current recommendations support the use of hydrocortisone at a physiological dose (i.e. 200-300 mg hydrocortisone $\cdot$ day $^{-1}$ ). These recommendations were based on the encouraging results of four small trials and subsequently one larger trial [19-23]. All five trials reported fewer deaths in patients who received GCs. A meta-analysis of these trials suggested that the use of corticosteroids reduced mortality [24]. As the balance of evidence regarding GC treatment for septic shock shifted towards the positive, a very important investigation, published in 2008, brought more shadows than light. This study represents the largest multicentric randomised placebocontrolled trial conducted on this topic to date, involving 499 patients with septic shock. The main result of this study was that hydrocortisone at a physiological dose does not decrease mortality in a general population of patients with septic shock, even though the drug hastens reversal of shock [25]. All in all, substantial uncertainty regarding the role of GCs in septic shock persists, and some authorities in the field have encouraged the launch of new trials with much higher numbers of patients in order to avoid generating further uncertainty [26].

The use of GCs as an adjunctive therapy in severe pneumonia has not been evaluated so extensively as in septic shock. A pilot study by MONTón et al. [27], in patients with severe pneumonia requiring mechanical ventilation, detected a possible immunosuppressive effect of GCs in pneumonia. In this study, a decrease in levels of pro-inflammatory cytokines, such as IL- 6 and TNF- $\alpha$, was observed in both serum and BALF from patients who had received GCs as a coadjuvant treatment (in most cases, as a bronchodilatory treatment 
associated with antibiotic treatment). Furthermore, in the group of patients receiving GC treatment, a trend towards lower mortality was also observed, although the population of the study comprised only 20 patients.

The relationship between the intensity of the inflammatory response in severe pneumonia, GC dosage and prognosis was subsequently studied by AGUSTí et al. [28]. In this study, the inflammatory response in BALF and serum from patients with severe pneumonia who had received GC treatment for long periods ( $>30$ days) was assessed. Results were compared to those from a group of patients with severe pneumonia without GC treatment and with those from a third group of patients with pneumonia who had received GC treatment for a short period of time $(9 \pm 7$ days, in most cases as a bronchodilatory treatment). These authors observed that the local inflammatory response (in BALF) and systemic inflammatory response (in serum), measured by such relevant cytokines as IL- 6 or TNF- $\alpha$, was markedly diminished in patients who had received GCs for longer periods of time compared to those who had not received such treatment. Furthermore, short-term administration of GCs had an intermediate effect in the suppression of the inflammatory response.

The mortality of the group of patients with severe pneumonia who had received long-term GC treatment was similar to that of patients who had not received GC treatment. Interestingly, the patients who had received GC treatment for short periods of time and had shown an attenuated inflammatory response exhibited a tendency towards lower mortality [28].

These results suggest that deep attenuation of the inflammatory response by prolonged corticosteroid treatment can be as harmful as an exaggerated inflammatory response, but its moderated attenuation by a short corticosteroid treatment can be beneficial for the modulation of the inflammatory response and for the prognosis of the disease. Studies performed by $\mathrm{KEH}$ et al. [29] seem to confirm the beneficial effects of low-dose GC treatment. Those authors demonstrated that low dosages of hydrocortisone achieved rapid haemodynamic stabilisation in patients with septic shock. The study verified that hydrocortisone attenuates the inflammatory and anti-inflammatory response, but does not have a negative effect on the phagocytic function of macrophages and monocytes.

In another work by IOANAS et al. [5], in a series of patients with ICU-acquired pneumonia requiring mechanical ventilation, the authors found that high levels of IL- 6 and IL-8 at the time of diagnosis and IL-6 levels on day 3 were the only factors related to the lack of response to empirical antibiotic treatment, suggesting, again, that modulation of the potential inflammatory response could also be beneficial in this subgroup of patients. Furthermore, on multivariate analysis of the several factors that could be related to the lack of response to treatment, the authors found that the concomitant administration of GCs was a protective factor (odds ratio (OR) 0.21).

MEDURI et al. [30] investigated prolonged methylprednisolone infusion $\left(1 \mathrm{mg} \cdot \mathrm{kg}\right.$ body weight $\left.{ }^{-1} \cdot \mathrm{day}^{-1}\right)$ in a randomised trial in 91 patients with early acute respiratory distress syndrome (ARDS), 43\% caused by severe CAP. Methylprednisolonetreated patients with severe $\mathrm{CAP}$ showed a higher rate of extubation (61 versus 14\%; $\mathrm{p}=0.07$ ) and lower $\mathrm{C}$-reactive protein levels $\left(2.5 \pm 1.8\right.$ versus $\left.12.1 \pm 8.1 \mathrm{mg} \cdot \mathrm{dL}^{-1} ; \mathrm{p}=0.06\right)$ by day 7. Treatment was associated with a nonsignificant reduction in the median duration of mechanical ventilation ( 5 versus 10 days; $\mathrm{p}=0.13$ ) and hospital mortality (16.5 versus $42.5 \% ; \mathrm{p}=0.3)$ [30].

Another randomised study by ANNANE et al. [21] in patients with septic shock showed that 28-day mortality among patients with severe CAP was 45 and $65 \%$ for patients randomised to low-dose hydrocortisone $(n=47)$ and placebo $(\mathrm{n}=54)$, respectively (OR $0.44 ; 95 \%$ confidence interval $(\mathrm{CI})$ $0.20-0.98 ; \mathrm{p}=0.044)$.

Finally, a recent retrospective study in 308 patients with severe CAP (defined as classes IV and V of the prognostic severity index score) showed that mortality was decreased in those patients who had received simultaneous administration of systemic corticosteroids along with antibiotic treatment (OR 0.28 ; 95\% CI 0.113-0.732) [31].

The only randomised controlled study in humans regarding the role of GCs in severe pneumonia was published in 2005 by CONFALONIERI et al. [32]. These authors assessed the efficiency and safety of the administration of a continuous infusion of hydrocortisone in a double-blind randomised placebocontrolled trial including 46 patients with severe CAP requiring ICU admission. Of these patients, 23 received an intravenous bolus of hydrocortisone at a dose of $200 \mathrm{mg}$ followed by a perfusion of $10 \mathrm{mg} \cdot \mathrm{h}^{-1}$ for 7 days. These authors demonstrated a mortality reduction in the group treated with hydrocortisone ( 0 versus $30 \% ; p=0.009$ ), a better modulation of the systemic inflammatory response (determined by serum Creactive protein level) and a significant improvement in the main clinical end-points, such as chest radiographic results, multiple organ dysfunction score (severity scale), arterial oxygen tension $\left(\mathrm{Pa}_{\mathrm{a}} \mathrm{O}_{2}\right)$ /inspiratory oxygen fraction $\left(\mathrm{FI}, \mathrm{O}_{2}\right)$ ratio, and duration of ICU and hospital stay.

Although the intimal mechanisms by which the inflammatory response modulated by GCs can interfere with pulmonary infection are not well elucidated, in vitro studies and experimental work in animals have provided some clues regarding this issue. The present authors' group has developed an experimental model of Pseudomonas aeruginosa pneumonia in ventilated pigs [10], in which the effect of GCs has been studied by comparing three groups of animals with severe pneumonia (those without treatment, those with antibiotic treatment and those with antibiotic treatment plus GCs). In this model, pigs treated with antibiotics plus GCs experienced, after 96 h of pneumonia onset, a decrease in the local inflammatory response compared to the other groups. Furthermore, animals treated with antibiotics plus GCs presented lower bacterial counts in both BALF and pulmonary tissue obtained at the end of the study, a finding that was related to a tendency to suffer from less-severe lesions, as revealed by a histopathological study [33]. In this sense, MEDURI et al. [34] have demonstrated, in an in vitro study, that certain bacterial strains possess receptors for the cytokines IL-1 $\beta$ and TNF- $\alpha$, and that the exposure of bacteria to these cytokines enhances their growth and virulence. It has been proposed that GCs might restore the impaired capacity of phagocytic cells produced by excessive inflammation. Exposing human monocytic (U937) cells to progressively higher concentrations of 
lipopolysaccharide (LPS) enhanced the intracellular survival and replication of various species of bacteria. More importantly, when exposed to graded concentrations of methylprednisolone, U937 cells previously stimulated with LPS were able to suppress bacterial replication efficiently in a concentration-dependent manner. Finally, TNF- $\alpha$, IL- $1 \beta$ and IL- 6 mRNA levels in LPSactivated cells were reduced by treatment of such cells with methylprednisolone [34].

\section{LESSONS TO BE LEARNED FROM SEPSIS AND ACUTE RESPIRATORY DISTRESS SYNDROME}

Although the previously mentioned findings seem to point towards a beneficial effect of GC treatment in severe pneumonia, definitive confirmation of these potential benefits is required. In this sense, many lessons can be learned from studies performed in patients with septic shock and ARDS.

The first studies conducted in patients with sepsis and pneumonia suggested a rapid improvement of symptoms in patients receiving GCs, although effects on mortality were not evidenced, and some cases were associated with a higher number of side-effects and with poorer prognosis once the steroid treatment was suspended [20]. Indeed, the potential for harmful side-effects due to GC treatment in these patients and the rebound effects that their tapering can cause in the evolution of the inflammatory process are the subject of intense debate [35]. The prolonged use of GCs can alter the phagocytic action of macrophages and alveolar granulocytes, which can facilitate the acquisition of severe bacterial and opportunistic infections [36]. Several studies involving patients with sepsis and ARDS have suggested that high-dose GCs increase the risk of secondary infections [37-39]; however, a meta-analysis of moderate-dose GCs for sepsis did not substantiate this observation [40]. The randomised controlled trial promoted by the National Heart, Lung, and Blood Institute (Bethesda, MD, USA), which included 188 patients with ARDS, did not detect an increased rate of nosocomial infection in the methylprednisolone group [41]. Indeed, in this study, there were more cases of nosocomial pneumonia, septic shock and positive blood culture in the placebo group. The ARDS Network protocol did not incorporate infection surveillance, making it impossible to estimate the impact of undiagnosed infections on outcome. Failed or delayed recognition of nosocomial infections in the presence of a blunted febrile response represents a serious threat and may be more common than initially suspected. In the two randomised trials that incorporated infection surveillance [30,42], nosocomial infections were frequently $(56 \%)$ identified in the absence of fever.

Another important aspect to bear in mind is the duration of suppression of the GC treatment. There is ample evidence that rapid tapering of GC treatment can induce a haemodynamic and immunological rebound effect if pro-inflammatory cytokine levels increase again and their receptors continue being suppressed [43]. Studies conducted in patients with septic shock have demonstrated that hydrocortisone infusion produces a significant decrease in the circulating levels of proteins dependent upon the transcription factor nuclear factor- $\kappa \mathrm{B}$, such as phospholipase $A_{2}$, IL-6 and -8 , and soluble E-selectin [29]. The suppression of the treatment provokes a rebound effect in most of these mediators, which highlights the shortacting anti-inflammatory action of hydrocortisone and the need to provide prolonged treatment in order to achieve a durable anti-inflammatory effect $[29,44]$. As acknowledged by the authors of the ARDS Network trial, rapid tapering and removal of methylprednisolone probably contributed to the deterioration in $\mathrm{Pa}_{1} \mathrm{O}_{2} / \mathrm{FI}, \mathrm{O}_{2}$ ratio and higher rate of intubation in the treatment arm [41].

Another concerning issue is the influence of GCs on muscle function. Certainly, the effects of moderate-dose GCs in crtically ill patients are now well understood. Recent studies of patients receiving assisted ventilation have found a strong association between corticosteroid treatment and muscle weakness $[45,46]$. In the ARDS Network trial, the overall rate of clinically suspected neuromyopathy was similar in the two groups evaluated; however, all nine reports of serious adverse events related to neuropathy or myopathy were in patients treated with methylprednisolone [41].

\section{SUMMARY}

In summary, there is evidence of efficacy of GCs in severe infections including severe pneumonia. Prolonged GC treatment in sepsis and ARDS is uniformly associated with significant improvement in various physiological and clinical parameters, such as improvement in $\mathrm{Pa}, \mathrm{O}_{2} / \mathrm{FI}_{1} \mathrm{O}_{2}$ ratio, hastened reversal of shock and significant reduction in levels of markers of systemic inflammation and duration of mechanical ventilation and ICU stay [47].

In severe pneumonia, scientific evidence is still scarce. Current information suggests that treatment with low dosages of GC is able to modulate (or diminish) the inflammatory response associated with pneumonia and improve prognosis in this disease, mainly in cases in which an increased host inflammatory response has been demonstrated.

Although the only randomised controlled trial of glucocorticosteroids in severe pneumonia showed an improvement in mortality, lessons learned from sepsis and acute respiratory distress syndrome impose the exercise of considerable caution. Reliable treatment recommendations will be possible only if a much larger trial is conducted and many different key points are considered. It is of the utmost importance that studies are designed not only to give a better understanding of the effect of glucocorticosteroids in severe pneumonia but also to determine the type of glucocorticosteroid (hydrocortisone or methylprednisolone), the dosage to be administered and the duration and tapering of the treatment. A series of preventative measures must be implemented in the design of new trials in order to avoid severe harmful secondary effects, including: 1) intensive infection surveillance, 2) avoidance of paralytic agents, 3) avoidance of rebound inflammation with premature discontinuation of treatment, and 4) strict control of hyperglycaemia.

\section{REFERENCES}

1 Alvarez-Lerma F, Torres A. Severe community-acquired pneumonia. Curr Opin Crit Care 2004; 10: 369-374.

2 Leeper KV, Torres A. Community-acquired pneumonia in the intensive care unit. Clin Chest Med 1995; 16: 155-171.

3 Heyland DK, Cook DJ, Griffith L, Keenan SP, BrunBruisson $\mathrm{C}$. The attributable morbidity and mortality of ventilator-associated pneumonia in the critically ill patient. Am J Respir Crit Care Med 1999; 159: 1249-1256. 
4 Menéndez $\mathrm{R}$, Torres $\mathrm{A}$, Rodriguez de Castro $\mathrm{F}$, et al. Predictive factors of clinical stability in communityacquired pneumonia. Thorax 2004; 59: 960-965.

5 Ioanas M, Ferrer M, Cavalcanti M, et al. Causes and predictors of nonresponse to treatment of ICU-acquired pneumonia. Crit Care Med 2004; 32: 938-945.

6 Sibille Y, Reynolds HY. Macrophages and polymorphonuclear neutrophils in lung defense and injury. Am Rev Respir Dis 1990; 141: 471-501.

7 Nelson S, Bagby GJ, Bainton BG, Wilson LA, Thompson JJ, Summer WR. Compartimentalization of intraalveolar and systemic lipopolysaccharide-induced tumor necrosis factor and the pulmonary inflammatory response. I Infect Dis 1989; 159: 189-194.

8 Martin C, Saux P, Mege JL, Perrin G, Papazian L, Gouin F. Prognostic value of serum cytokines in septic shock. Intensive Care Med 1994; 20: 272-277.

9 Dehoux MS, Boutten A, Ostinelli J, et al. Compartmentalized cytokine production within the human lung in unilateral pneumonia. Am J Respir Crit Care Med 1994; 150: 710-716.

10 Sibila O, Agusti C, Torres A, et al. Experimental Pseudomonas aeruginosa pneumonia: evaluation of the associated inflammatory response. Eur Respir I 2007; 30; 1167-1172.

11 Puren AJ, Feldman C, Savage N, Becker PJ, Smith C. Patterns of cytokine expression in community-acquired pneumonia. Chest 1995; 107: 1342-1349.

12 Monton C, Torres A, El-Ebiary M, et al. Cytokine expression in severe pneumonia: a bronchoalveolar study. Crit Care Med 1999; 27: 1745-1753.

13 Fernandez-Serrano S, Dorca J, Coromines M, Carratalà J, Gudiol F, Manresa F. Molecular inflammatory response measured in blood of patients with severe community-acquired pneumonia. Clin Diag Lab Immunol 2003; 10: 813-820.

14 Yende S, Tuomanen EI, Wunderink RG, et al. Pre-infection systemic inflammatory markers and risk of hospitalisation due to pneumonia. Am J Respir Crit Care Med 2005; 172; 535-541.

15 De Bosscher K, Vanden Berghe W, Haegeman G. The interplay between the glucocorticoid receptor and nuclear factor $-\kappa \mathrm{B}$ or activator protein-1: molecular mechanisms for gene repression. Endocr Rev 2003; 24: 488-522.

16 Rhen T, Cidlowsky JA. Antiinflammatory action of glucocorticoids - new mechanisms for old drugs. $N$ Engl J Med 2005; 353: 1711-1723.

17 Hafezi-Moghadam A, Simoncini T, Yang Z, et al. Acute cardiovascular protective effects of corticosteroids are mediated by non-transcriptional activation of endothelial nitric oxide synthase. Nat Med 2002; 8: 473-479.

18 Briel M, Boscacci R, Furrer H, et al. Adjunctive corticosteroids for Pneumocystis jiroveci pneumonia in patients with HIV infection: a meta-analysis of randomized controlled trials. BMC Infect Dis 2005; 5: 101.

19 Bollaert PE, Charpentier C, Levy B, Debouverie M, Audibert G, Larcan A. Reversal of late septic shock with supraphysiologic doses of hydrocortisone. Crit Care Med 1998; 26: 645-650.

20 Briegel J, Forst $\mathrm{H}$, Haller $\mathrm{M}$, et al. Stress doses of hydrocortisone reverse hyperdynamic septic shock: a prospective, randomized, double-blind, single-center study. Crit Care Med 1999; 27: 723-732.
21 Annane D, Sébille V, Charpentier C, et al. Effect of treatment with low doses of hydrocortisone and fludrocortisone on mortality in patients with septic shock. JAMA 2002; 288; 862-871.

22 Dellinger RP, Carlet JM, Masur H, et al. Surviving sepsis campaign guidelines for management of severe sepsis and septic shock. Crit Care Med 2004; 32; 858-873.

23 Minecci PC, Deans KJ, Banks SM, et al. Meta-analysis: the effect of steroids on survival and shock during sepsis depends on the dose. Ann Intern Med 2004; 141: 47-56.

24 Annane D, Bellisant E, Bollaert PE, et al. Corticosteroids for severe sepsis and septic shock: a systematic review and meta-analysis. BMJ 2004; 329: 480.

25 Sprung CL, Annane D, Keh D, et al. Hydrocortisone therapy for patients with septic shock. N Engl J Med 2008; 358: 111-124.

26 Finfer S. Corticoesteroids in septic shock. $N$ Engl J Med 2008; 358: 188-190.

27 Montón C, Ewig S, Torres A, et al. Role of glucocorticoids on inflammatory response in nonimmunosuppressed patients with pneumonia: a pilot study. Eur Respir J 1999; 14: $218-220$

28 Agustí C, Rañó A, Filella X, et al. Pulmonary infiltrates in patients receiving long-term glucocorticoid treatment. Etiology, prognostic factors and associated inflammatory response. Chest 2003; 123: 488-498.

29 Keh D, Boenhke T, Weber-Cartens S, et al. Immunologic and hemodynamic effects of "low-dose" hydrocortisone in septic shock. A double blind, randomised, placebocontrolled, crossover study. Am J Respir Crit Care Med 2003; 167: 512-520.

30 Meduri GU, Golden E, Freire AX, et al. Methylprednisolone infusion in early acute severe ARDS: results of a randomized controlled trial. Chest 2007; 131: 954-963.

31 Garcia-Vidal C, Calbo E, Pascual V, Ferrer C, Quintana S, Garau J. Effects of systemic steroids in patients with severe community-acquired pneumonia. Eur Respir J 2007; 30: 951-956.

32 Confalonieri M, Urbino G, Potena A, et al. Hydrocortisone infusion for severe community-acquired pneumonia. A preliminary randomized study. Am J Respir Crit Care Med 2005; 171: 242-248.

33 Sibila O, Luna CM, Agustí C, et al. Effects of glucocorticoids in ventilated piglets with severe pneumonia. Eur Respir J 2008; [Epub ahead of print PMID: 18508831].

34 Meduri GU, Kanangat S, Bronze M, et al. Effects of methylprednisolone on intracellular bacterial growth. Clin Diagn Lab Immunol 2001; 8: 1156-1163.

35 Lefering R, Neugebauer EM. Steroid controversy in sepsis and septic shock: a meta-analysis. Crit Care Med 1995; 23: 1294-1303.

36 Meersseman W, Vandecasteele SJ, Wilmer A, Verbeken E, Peetermans WE, Van Wijngaerden E. Invasive aspergillosis in critically ill patients without malignancies. Am J Respir Crit Care Med 2004; 170; 621-625.

37 Bernard GR, Luce JM, Sprung CL, et al. High-dose corticosteroids in patients with the adult respiratory distress syndrome. N Engl J Med 1987; 317: 1565-1570.

38 Weigelt JA, Norcross JF, Borman KR, Snyder WH 3rd. Early steroid therapy for respiratory failure. Arch Surg 1985; 120: 536-540. 
39 Sprung CL, Caralisis PV, Marcial EH, et al. The effects of high-dose corticosteroids in patients with septic shock. A prospective, controlled study. N Engl J Med 1984; 311: 1137-1143.

40 Cronin L, Cook DJ, Carlet J, et al. Corticoesteroid treatment for sepsis: a critical appraisal and meta-analysis of the literature. Crit Care Med 1995; 23: 1430-1439.

41 Steinberg KP, Hudson LD, Goodman RB, et al. Efficacy and safety of corticosteroids for persistent acute respiratory distress syndrome. N Engl J Med 2006; 354: 1671-1684.

42 Meduri GU, Headley S, Golden E, et al. Effect of prolonged methylprednisolone therapy in unresolving acute respiratory distress syndrome. A randomized controlled trial. JAMA 1998; 280: 159-165.

43 Barber AE, Coyle SM, Fischer E. Influence of hypercortisolemia on soluble tumor necrosis factor receptor II and interleukin-1 receptor antagonist responses to endotoxin in human beings. Surgery 1995; 118: 406-410.

44 Briegel J, Kellermann W, Forst H, et al. Low-dose hydrocortisone infusion attenuates the systemic inflammatory response syndrome. Clin Invest 1994; 72: 782-787.

45 Herrige MS, Cheung AM, Tansey CM, et al. One-year outcomes in survivors of the acute respiratory distress syndrome. N Engl J Med 2003; 348: 683-693.

46 De Jonghe B, Sharshar T, Lefaucheur JP, et al. Paresis acquired in the intensive care unit: a prospective multicenter study. JAMA 2002; 288: 2859-2867.

47 Meduri GU, Marik P, Chrousos GP, et al. Steroid treatment in ARDS; a critical appraisal of the ARDS network trial and the recent literature. Intensive Care Med 2008; 34: 61-69. 